\title{
MÉTODO AUTOMÁTICO PARA CONTAR VAGENS DE AMENDOIM ATRAVÉS DE IMAGEM RGB
}

Adão Felipe dos Santos ${ }^{1}$; Danilo Tedesco de Oliveira ${ }^{2}$; Rouverson Pereira da Silva ${ }^{3}$; Cristiano Zerbato $^{4}$; Walter Maldonado $\mathrm{Jr}^{5}$

'Doutorando em Agronomia (Produção Vegetal), FCAV/UNESP, Jaboticabal, SP, adaofeliped@gmail.com; ${ }^{2}$ Mestrando em Agronomia (Produção Vegetal), FCAV/UNESP, Jaboticabal, SP; ${ }^{3}$ Docente da FCAV/UNESP, Jaboticabal, SP; ${ }^{4}$ Docente da FCAV/UNESP, Jaboticabal, SP; ${ }^{5}$ Docente da FCAV/UNESP, Jaboticabal, SP.

RESUMO: O desenvolvimento de ferramentas que automatizam os processos agrícolas tem ganhado espaço nos últimos tempos, principalmente pela introdução de ferramentas computacionais que auxiliam os produtores nas tomadas de decisão. Dessa forma, objetivou-se desenvolver um algoritmo para identificar vagens de amendoim através de imagens RGB. As imagens foram capturadas usando um smartphone com e sem o uso do flash em uma altura aproximadamente de $40 \mathrm{~cm}$, com 10 repetições para cada condição. A imagem de entrada foi em RGB, utilizou no processamento a imagem na banda R. As imagens foram limiarizadas para que as vagens de amendoim fossem destacadas, de modo a eliminar o solo da imagem. Em seguida aplicou-se o comando para dilatar, reduzir ruídos e por fim contar o número de vagens de acordo com o tamanho de pixel $(0,30,60,90$ e 120). Os melhores resultados foram encontrados quando o flash estava ativado em conjunto com a função de pixel maior que 60. O algoritmo foi capaz de detectar as vagens de amendoim e contar com boa acurácia. Acredita-se que é possível estimar as perdas e produtividade de amendoim por meio de imagem RGB a partir do algoritmo desenvolvido.

Palavras-chave: Machine learning, estimativa de produtividade, agricultura de precisão.

\section{INTRODUÇÃO}

A análise de imagens é uma importante área de pesquisa no domínio agrícola que utiliza-se de técnicas inteligentes para análise de dados, identificação e classificação de imagens, detecção de anomalias, dentre outras diversas aplicações agrícolas (SINGH et al., 2016; KAMILARIS; PRENAFETA-BOLDÚ, 2018). Dentre as técnicas mais populares usadas para analisar imagens incluem o aprendizado de máquina, popularmente conhecido como "machine learning”, em que pode-se empregar um dos métodos de aprendizagem (K-means, máquinas de vetores de suporte, redes neurais artificiais) para se obter bons resultados na análise das imagens (SAXENA; ARMSTRONG, 2014; SINGH et al., 2016).

A utilização de imagens RGB, associada a aprendizado de máquina, foi relatada no Brasil com boa acurácia por MALDONADO \& BARBOSA (2016) na estimativa de contagem de frutos de laranja. Dessa forma, pressupõem-se que é possível utilizar técnicas de aprendizado de máquina para 
16 e 17 de agosto de 2018, centro de convenções da FCAV/UNESP - Câmpus de Jaboticabal, SP

identificar e contar as vagens de amendoim e posteriormente estimar as perdas na colheita e a produtividade da cultura.

Para os produtores de amendoim essa técnica pode ter boa aplicação, visto que para a cultura ainda não é reportado nenhum método de previsão de produtividade utilizando aprendizado de máquina, muito menos tecnologia embarcada nas máquinas utilizadas nas operações de colheita (arranquio e recolhimento). Este fato, aliado a baixa precisão na estimativa de maturação, faz com que os produtores de amendoim brasileiros não tenham retornos substancialmente satisfatórios, uma vez que a gestão da colheita é basicamente regulada por esses dois parâmetros.

Dessa forma, este trabalho propõem um método não-destrutivo de identificar e classificar de forma automatizada as vagens de amendoim, e em caso de boa acurácia, propor um novo método de estimativa de perdas e produtividade de amendoim por meio de imagens digitais.

\section{MATERIAL E MÉTODOS}

As vagens utilizadas para captura das imagens foram provenientes de uma área de produção comercial do cultivar IAC OL3, sendo que essas vagens foram quantificadas como perdas durante a operação de arranquio e foram coletadas em $2 \mathrm{~m}^{2}$.

As imagens foram capturadas utilizando um smartphone modelo iphone $5 \mathrm{~s}$ sob duas configurações: Com o flash ligado e desligado, em uma altura aproximadamente de $40 \mathrm{~cm}$, com 10 repetições para cada condição. No processamento das imagens, testaram-se, no software, cinco tamanhos de pixel para verificar qual seria o melhor para processar as imagens de forma automática com maior probabilidade de acerto na estimativa das vagens. $O$ roteiro do processamento das imagens foi conforme a Figura 1.

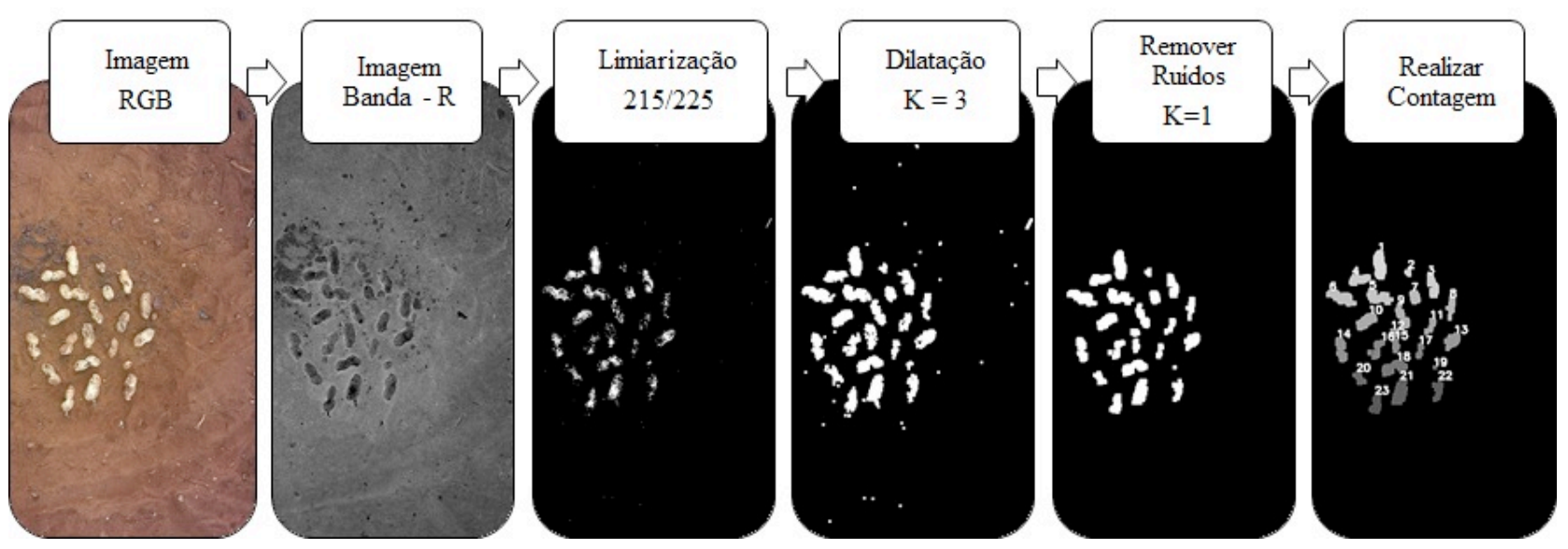

Figura 1. Roteiro para obtenção do número de vagens pelo método automático desenvolvido em Python.

Todos os procedimentos de processamento de imagens utilizados neste trabalho foram realizados em Python fazendo uso da biblioteca de visão computacional OpenCV (BRADSKI, 2018). 
As vagens foram colocadas sobre a superfície do solo para simular uma condição de campo. Ressalta-se que as vagens utilizadas haviam sido previamente limpas e secadas em estufas para posterior correção de $8 \%$ de umidade.

Por fim, para verificar a relação entre o número de vagens visíveis nas imagens e o número de vagens contadas pelo algoritmo, utilizou-se análise de regressão linear, sendo a confiabilidade dada pelo valor de ajuste $\mathrm{R}^{2}$, também realizou-se estatística descritiva para verificar o comportamento dos dados e identificar qual o valor do tamanho de pixel, que se aproximava mais da contagem manual.

\section{RESULTADOS E DISCUSSÃO}

Em relação a captura das imagens os resultados mostraram que quando se utilizou o flash do celular o algoritmo conseguiu melhorar a acurácia na contagem das vagens. Notou-se ainda melhoria na acurácia de contagem quando se utilizou o valor do pixel para contagem de objetos maior do que 60. Dessa forma, a escolha do tamanho do pixel para o modelo de predição automática foi embasada pelos valores máximo e mínimo serem iguais ao da contagem manual e ainda pelo fato de que usando esse tamanho de pixel no algoritmo foi quando se obteve uma média mais próxima da contagem manual (Tabela 1).

Tabela 1. Estatística descritiva para a contagem automática de vagens de amendoim com o flash ligado

\begin{tabular}{ccccccc} 
& & \multicolumn{5}{c}{ Contagem automática das vagens com flash } \\
\cline { 2 - 6 } & $\begin{array}{c}\text { Contagem } \\
\text { manual }\end{array}$ & 0 & 30 & $\mathbf{6 0}$ & 90 & 120 \\
\hline Média & $\mathbf{2 0 , 2}$ & 23 & 22,2 & $\mathbf{1 9 , 2}$ & 17,3 & 16 \\
Máx & $\mathbf{2 7}$ & 45 & 42 & $\mathbf{2 7}$ & 26 & 25 \\
Min & $\mathbf{1 2}$ & 12 & 12 & $\mathbf{1 2}$ & 12 & 12 \\
Desv P. & $\mathbf{5 , 0 6}$ & 8,97 & 8,35 & $\mathbf{4 , 9 6}$ & 4,38 & 4,20 \\
CV \% & $\mathbf{2 5 , 0 2}$ & 38,98 & 37,62 & $\mathbf{2 5 , 8 1}$ & 25,33 & 26,22 \\
\hline
\end{tabular}

Com base no modelo de regressão linear, encontrou-se pouca diferença entre os valores observados e os estimados (Figura 2). Na maioria das imagens analisadas o método automático foi capaz de estimar com boa acurácia a quantidade de vagens de amendoim que estavam sobre o solo, o que indica que é possível de se obter a partir de imagens digitais a contagem automática de vagens de amendoim e consequentemente estimar as perdas e a produtividade de amendoim. 


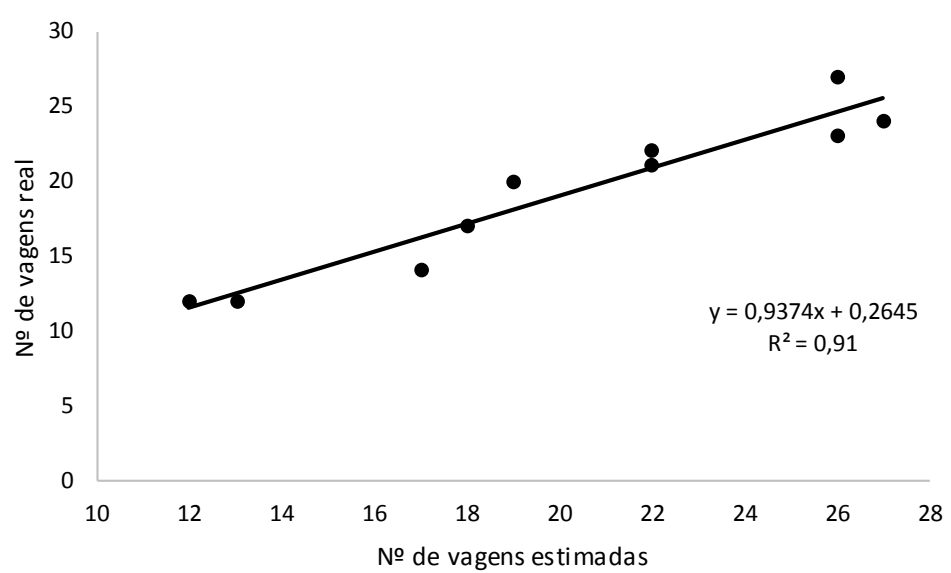

Figura 2. Regressão linear para a relação entre o número de vagens contadas pelo algoritmo $\left(\mathrm{n}^{\mathrm{o}} \mathrm{de}\right.$ vagens estimadas) e as contadas manualmente ( $\mathrm{n}^{\mathrm{o}}$ de vagens real)

Quanto a previsão do peso das amostras, com base no número de vagens estimadas pelo método automático, pode-se dizer que a estimativa de peso é confiável, visto que o ajuste da regressão foi de $\mathrm{R}^{2}=0,95$ com erro médio de $\pm 1,6$ gramas (Figura 3 ). Do ponto de vista técnico-prático a previsão precisa da produtividade aliado ao uso de tecnologias podem ajudar os agricultores a melhorar a qualidade das operações (KAMILARIS \& PRENAFETA-BOLDÚ, 2018), principalmente no caso do amendoim que demanda duas operações para a colheita, fazendo com que eles tomem melhores decisões sobre a intensidade da colheita, tais como ajustes nas máquinas de acordo com o potencial produtivo de cada área.

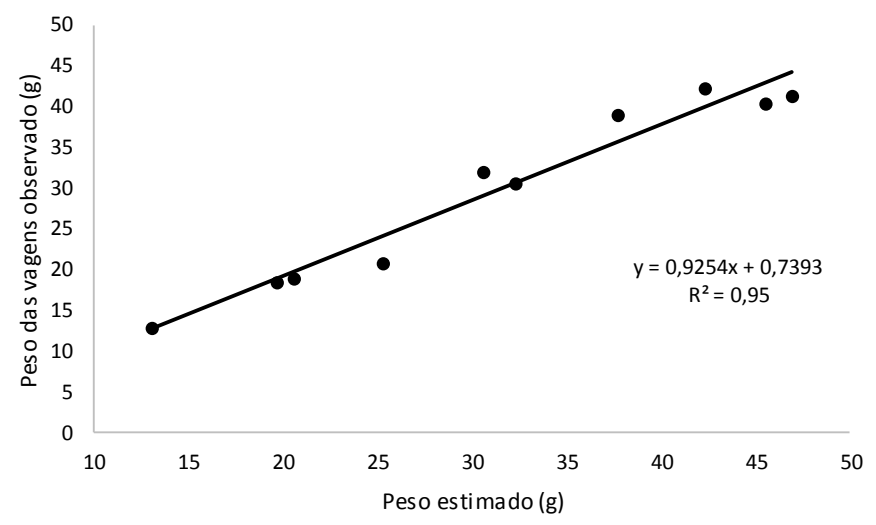

Figura 3. Regressão linear para o peso das amostras estimado pelo algoritmo e do peso real observado

Especificamente na cultura do amendoim nota-se atrasos na tecnologia embarcada das máquinas, sendo que as colhedoras não possuem sensores de produtividades como as de outras culturas, o que dificulta o produtor saber a produtividade em tempo real no momento da colheita. Com os bons resultados de estimativa encontrados neste trabalho os produtores de amendoim poderão em breve experimentar estimar a produtividade de suas lavouras por meio de imagem digital. 
Entretanto, um dos fatores que podem interferir na acurácia da estimativa está na quantidade de material vegetal presente no solo, além disso vale ressaltar que nas condições experimentais não havia sobreposição de vagens, o que pode interferir na estimativa usando imagem digital. RAHNEMOONFAR \& SHEPPARD (2017), encontraram modelos que permitem contar com confiabilidade frutos que possuem formatos circulares, porém, além de imagens digitais os autores utilizaram rede neurais artificiais, que tem capacidade de aprendizado a medida em que essa executa o trabalho.

\section{CONCLUSÃO}

O algoritmo de contagem automática de vagens de amendoim utilizando imagens RGB possui boa acurácia na estimativa do número de vagens, bem como do peso médio das amostras, o que indica potencial uso na estimativa de perdas de produtividade em amendoim.

\section{AGRADECIMENTOS}

Os autores prestam seus agradecimentos ao Conselho Nacional de Desenvolvimento Científico e Tecnológico (CNPq).

\section{REFERÊNCIAS BIBLIOGRÁFICAS}

BRADSKI, G., 2018. Open Source Computer Vision Library [https:/github.com/opencv/opencv]. GitHub. (acessado em 28/6/18)

KAMILARIS, A., PRENAFETA-BOLDÚ, F.X. Deep learning in agriculture: A survey. Computers and Electronics in Agriculture, 147, 70-90, 2018.

MALDONADO JR, W., BARBOSA, J.C. Automatic green fruit counting in orange trees using digital images. Computers and Electronics in Agriculture, 127, 572-581, 2016.

RAHNEMOONFAR, M., SHEPPARD, C. Deep count: fruit counting based on deep simulated learning. Sensors 17 (4), 905, 2017.

SINGH, A., GANAPATHYSUBRAMANIAN, B., SINGH, A.K., SARKAR, S. Machine learning for high-throughput stress phenotyping in plants. Trends Plant Sci. 21, 110-124, 2016.

SAXENA, L., ARMSTRONG, L. A survey of image processing techniques for agriculture. Proceedings of Asian Federation for Information Technology in Agriculture, Australian Society of Information and Communication Technologies in Agriculture. Perth, Austrália, 2014. 